\title{
Atomic Broadcast in Asynchronous Crash-Recovery Distributed Systems
}

\author{
Luís Rodrigues \\ Michel Raynal
}

DI-FCUL

TR-99-7

Departamento de Informática

Faculdade de Ciências da Universidade de Lisboa

Campo Grande, 1749-016 Lisboa

Portugal

Technical reports are available at http://www.di.fc.ul.pt/biblioteca/tech-reports. The files are stored in PDF, with the report number as filename. Alternatively, reports are available by post from the above address. 



\title{
Atomic Broadcast in Asynchronous Crash-Recovery Distributed Systems*
}

\author{
Luís RODRIGUES \\ Universidade de Lisboa \\ FCUL, Campo Grande, \\ 1749-016 Lisboa, Portugal \\ ler@di.fcul.pt
}

\author{
Michel RAYNAL \\ IRISA \\ Campus de Beaulieu \\ 35 042 Rennes-cedex, France \\ raynal@irisa.fr
}

\begin{abstract}
Atomic Broadcast is a fundamental problem of distributed systems: it states that messages must be delivered in the same order to their destination processes. This paper describes a solution to this problem in asynchronous distributed systems in which processes can crash and recover.

A Consensus-based solution to the Atomic Broadcast problem has been designed by Chandra and Toueg for asynchronous distributed systems where crashed processes do not recover. Although our solution is based on different algorithmic principles, it follows the same idea: it transforms any Consensus protocol suited to the crash-recovery model into an Atomic Broadcast protocol suited to the same model. We show that Atomic Broadcast can be implemented without requiring any additional log operations in excess of those required by the Consensus. The paper also discusses how additional log operations can improve the protocol in terms of faster recovery and better throughput. Our work benefits from recent results in the study of the Consensus problem in the crash-recovery model.
\end{abstract}

Keywords: Distributed Fault-Tolerance, Asynchronous Systems, Atomic Broadcast, Consensus, Process Crash, Process Recovery.

\section{Introduction}

Atomic Broadcast is one of the most important agreement problems encountered in the design and in the implementation of fault-tolerant distributed systems. This problem consists in providing processes with a communication primitive that allows them to broadcast and deliver messages in such a way that processes agree not only on the set of messages they deliver but also on the order of message deliveries. Atomic Broadcast (sometimes called Total Order Broadcast) has been identified as a basic communication primitive in many systems (such as the ones described in [16]). It is particularly useful to implement fault-tolerant

\footnotetext{
* This report revises and expands parts of TR-99-1, "Atomic Broadcast and Quorum-based Replication in Asynchronous Crash-Recovery Distributed Systems".
} 
services by using software-based replication [8]. By employing this primitive to disseminate updates, all correct copies of a service deliver the same set of updates in the same order, and consequently the state of the service is kept consistent.

Solutions to the Atomic Broadcast problem in asynchronous systems prone to process crash (no-recovery) failures are now well known $[3,6,17]$. In this model, process crashes are definitive (i.e., once crashed, a process never recovers), so, a failed process is a crashed process. Unfortunately, the crash-no recovery model is unrealistic for the major part of applications. That is why, in this paper, we consider the more realistic crash-recovery model. In this model, processes can crash and later recover. We assume that when a process crashes (1) it loses the content of its volatile memory, and (2) the set of messages that has been delivered while it was crashed is also lost. This model is well-suited to feature real distributed systems that support user applications. Real systems provide processes with stable storage that make them to cope with crash failures. A stable storage allows a process to log critical data. But in order to be efficient, a protocol must not consider all its data as critical and must not log a critical data every time it is updated (the protocol proposed in this paper addresses these efficiency issues).

It has been shown in [3] that Atomic Broadcast and Consensus are equivalent problems in asynchronous systems prone to process crash (no-recovery) failures. The Consensus problem is defined in the following way: each process proposes an initial value to the others, and, despite failures, all correct processes have to agree on a common value (called decision value), which has to be one of the proposed values. Unfortunately, this apparently simple problem has no deterministic solution in asynchronous distributed systems that are subject to even a single process crash failure: this is the so-called Fischer-Lynch-Paterson's (FLP) impossibility result [5]. The FLP impossibility result has motivated researchers to find a set of minimal assumptions that, when satisfied by a distributed system, makes Consensus solvable in this system. The concept of unreliable failure detector introduced by Chandra and Toueg constitutes an answer to this challenge [3]. From a practical point of view, an unreliable failure detector can be seen as a set of oracles: each oracle is attached to a process and provides it with information regarding the status of other processes. An oracle can make mistakes, for instance, by not suspecting a failed process or by suspecting a not failed one. Although failure detectors were originally defined for asynchronous systems where processes can crash but never recover, the concept has been extended to the crash-recovery model $[1,4,11,14]$. The reader should be aware that the existing definitions of failure detectors in the former model do have quite significant differences.

Chandra and Toueg have also shown how to transform any Consensus protocol into an Atomic Broadcast protocol in the asynchronous crash (no-recovery) model [3]. In the present paper we follow a similar line of work and we show how to transform a protocol that solves Consensus in the crash-recovery model in a protocol that solves Atomic Broadcast in the same model. Thus, our protocol assumes a solution to the Consensus problem in the crash-recovery model (such protocols are described in $[1,4,11,14]])$. Our transformation owns several interesting properties. In the first place, it does not require the explicit use of failure detectors (although those are required to solve the Consensus problem). 
Thus, it is not bound to any particular failure detection mechanism. It relies on a gossip mechanism for message dissemination, avoiding the problem of reliable multicast in the crash-recovery model. Also, it allows recovering processes to skip over Consensus executions that already have a decided outcome. Additionally, our solution is non-blocking [2], i.e., as long as the system allows Consensus to terminate the Atomic Broadcast is live. Finally, but not the least, we show that Atomic Broadcast can be implemented without requiring any additional $\log$ operations in excess of those required by the Consensus. Thus, our protocol is optimal in terms of number of log operations.

Chandra-Toueg's approach and ours are similar in the sense that both of them transform a Consensus protocol into an Atomic Broadcast protocol. But, as they consider different models (crash-no recovery), they are based on very different algorithmic principles. This comes from the fact that we have to cope with process crashes and message losses (that is why our protocol requires a gossiping mechanism, which is not necessary in a crash-no recovery + no message loss model). Actually, when solving a distributed system problem, modifying the underlying system model very often requires the design of protocols suited to appropriate models [9].

The paper is organized as follows. Section 2 defines the crash-recovery model and the Atomic Broadcast problem in such a model. Then, Section 3 presents the underlying building blocks on top of which the proposed protocol is built, namely, a transport protocol and a Consensus protocol suited to the crashrecovery model. A minimal version of our Atomic Broadcast protocol for the crash-recovery model is then presented in Section 4. As indicated before, this protocol actually extends to the crash-recovery model the approach proposed by Chandra and Toueg for the crash-no recovery model [3]. The protocol proceeds in asynchronous rounds. Each round defines a delivery order for a batch of messages. This common delivery order is defined by solving an instance of the Consensus problem. The extension of this approach to the crash-recovery model is not trivial as (due to crashes) messages can be lost, and (due to crashes and recoveries) process states can also be lost. The impact of additional log operations on the protocol is discussed in Section 5. Section 6 relates the Atomic Broadcast problem in the crash-recovery with other relevant problems. Finally, Section 7 concludes the paper.

\section{Atomic Broadcast in the Crash-Recovery Model}

\subsection{The Crash-Recovery Model}

We consider a system consisting of a finite set of processes $\Pi=\{p, \ldots, q\}$. At a given time, a process is either up or down. When it is up, a process progresses at its own speed behaving according to its specification (i.e., it correctly executes its program text). While being up, a process can fail by crashing: it then stops working and becomes down. A down process can later recover: it then becomes up again and restarts by invoking a recovery procedure. So, the occurrence of the local event crash (resp. recover) generated by the local environment of a process, makes this process transit from up to down (resp. from down to up).

A process is equipped with two local memories: a volatile memory and a 
stable storage. The primitives log and retrieve allow an up process to access its stable storage. When it crashes, a process definitely loses the content of its volatile memory; the content of a stable storage is not affected by crashes.

Processes communicate and synchronize by sending and receiving messages through channels. We assume there is a bidirectional channel between each pair of processes. Channels are not necessarily FIFO; moreover, they can duplicate messages. Message transfer delays are finite but arbitrary. Even if channels are reliable, the combination of crashes, recoveries and arbitrary message transfer delays can entail message losses: the set of messages that arrive at a process while it is down are lost. Thus, the protocol must be prepared to recover from messages losses.

The multiplicity of processes and the message-passing communication makes the system distributed. The absence of timing assumptions makes it asynchronous. It is the role of upper layer protocols to make it reliable.

\subsection{Atomic Broadcast}

Atomic Broadcast allows processes to reliably broadcast messages and to receive them in the same delivery order. Basically, it is a reliable broadcast plus an agreement on a single delivery order. We assume that all messages are distinct. This can be easily ensured by adding an identity to each message, an identity being composed of a pair (local sequence number, sender identity)..

At the syntactical level, Atomic Broadcast is composed of two primitives: A-broadcast $(m)$ (used to send messages) and $\mu_{p}=\mathrm{A}$-deliver-sequence() (used by the upper layer to obtain the sequence of ordered messages). As in [3], when a process executes A-broadcast $(m)$ we say that it "A-broadcasts" $m$. We also define a boolean predicate A-delivered $\left(m, \mu_{p}\right)$ which evaluates to "true" is $m \in \mu_{p}$ or "false" otherwise. We also say that some process $p$ "A-delivers" $m$ if Adelivered $(m, \mathrm{~A}$-deliver-sequence ()$)$ is "true" at $p$.

In the context of asynchronous distributed systems where processes can crash and recover, the semantics ${ }^{1}$ of Atomic Broadcast is defined by the four following properties: Validity, Integrity, Termination and Total Order. This means that any protocol implementing these communication primitives in such a crash-recovery context has to satisfy these properties.

The validity property specifies which messages can be A-delivered by processes: it states that the set of A-delivered messages can not contain spurious messages.

- Validity: If a process A-delivers a message $m$, then some process has Abroadcast $m$.

The integrity property states there are no duplicates.

- Integrity: Let $\mu_{p}$ be the delivery sequence at a given process $p$. A message $m$ appears at most once in $\mu_{p}$.

The termination property specifies the situations where a message $m$ has to be A-delivered.

\footnotetext{
${ }^{1}$ We actually consider the definition of the Uniform Atomic Broadcast problem [10].
} 
- Termination: For any message $m,(1)$ if the process that issues A-broadcast $(m)$ eventually remains permanently up, or (2) if a process A-delivers a message $m$, then all processes that eventually remain up A-deliver $m$.

The total order property specifies that there is a single total order in which messages are A-delivered. This is an Agreement property that, joined to the termination property, makes the problem non-trivial.

- Total Order: Let $\mu_{p}$ be the sequence of messages A-delivered to process $p$. For any pair $(p, q)$, either $\mu_{p}$ is a prefix of $\mu_{q}$ or $\mu_{q}$ is a prefix of $\mu_{p}$.

\section{Underlying Building Blocks}

The protocol proposed in Section 4 is based on two underlying building blocks: a Transport Protocol and a protocol solving the Uniform Consensus problem. This section describes the properties and interfaces of these two building blocks.

\subsection{Transport Protocol}

The transport protocol allows processes to exchange messages. A process sends a message by invoking a send or multisend primitive ${ }^{2}$. Both send and multisend are unreliable: the channel can lose messages but it is assumed to be fair, i.e., if a message is sent infinitely often by a process $p$ then it is received infinitely often by its receiver [13]. When a message arrives at a process it is deposited in its input buffer that is a part of its volatile memory. The process will consume it by invoking a receive primitive. If the input buffer is empty, this primitive blocks its caller until a message arrives.

\subsection{Consensus Interface}

In the Consensus problem each process proposes a value and all correct processes have to decide on some value $v$ that is related to the set of proposed values [5]. The interface with the Consensus module is defined in terms of two primitives: propose and decided. As in previous works (e.g., [3]), when a process $p$ invokes $\operatorname{propose}(w)$, where $w$ is its proposal to the Consensus, we say that $p$ "proposes" $w$. A process proposes by logging its initial value on stable storage; this is the only logging required by our basic version of the protocol. In the same way, when $p$ invokes decided and gets $v$ as a result, we say that $p$ "decides" $v$ (denoted $\operatorname{decided}(v))$.

The definition of the Consensus problem requires a definition of a "correct process". This is done in Section 3.3. As the words "correct" and "faulty" are used with a precise meaning in the crash (no-recovery) model [3], and as, for clarity purpose, we do not want to overload them semantically, we define their equivalents in the crash-recovery model, namely, "good" and "bad" processes (we use the terminology of [1]). If crashed processes never recover, "good" and "bad" processes are equivalent with "correct" and "faulty" processes, respectively. Section 3.4 specifies the three properties defining the Consensus problem in this model.

\footnotetext{
${ }^{2}$ The primitive multisend is actually a macro that allows a process $p$ to send (by using the basic send primitive) a message to all processes (including itself).
} 


\subsection{Good and Bad Processes}

A good process is a process that eventually remains permanently up. A bad process is a process that is not good. So, after some time, a good process never crashes. On the other hand, after some time, a bad process either permanently remains crashed or permanently oscillates between crashes (down periods) and recoveries (up periods). From a practical point of view, a good process is a process that, after some time, remains up long enough to complete the upper layer protocol. In the Atomic Broadcast problem for example, this means that a good process that invokes A-broadcast $(m)$ will eventually terminate this invocation (it is possible that this termination occurs only after some (finite) number of crashes).

It is important to note that, when considering a process, the words "up" and "down" refer to its current state (as seen by an external observer), while the words "good" and "bad" refer to its whole execution.

\subsection{Consensus Definition}

The definition of the Consensus problem in the crash-recovery model is obtained from the one given in the crash (no-recovery) model by replacing "correct process" by "good process".

Each process $p_{i}$ has an initial value $v_{i}$ that it proposes to the others, and all good processes have to decide on a single value that has to be one of the proposed values. More precisely, the Consensus problem is defined by the following three properties (we actually consider the Uniform version [3] of the Consensus problem):

- Termination: Every good process eventually decides some value.

- Uniform Validity: If a process decides $v$, then $v$ was proposed by some process.

- Uniform Agreement: no two processes (good or bad) decide differently.

\subsection{Enriching the Model to Solve Consensus}

As noted previously, the Consensus problem has no deterministic solution in the simple crash (no-recovery) model. This model has to be enriched with a failure detector that, albeit unreliable, satisfies some minimal conditions in order that the Consensus be solvable.

In the same way, the crash-recovery model has to be augmented with a failure detector so that the Consensus can be solved. Different types of failure detectors have been proposed to solve the Consensus problem in the crash-recovery model. Protocols proposed in $[11,14]$ use similar failure detectors that outputs list of "suspects"; so, their outputs are bounded. [1] uses failure detectors whose outputs are unbounded (in addition to lists of suspects, the outputs include counters). The advantage of the later is that they do not require the failure detector to predict the future behavior of bad processes. A positive feature of our protocol is that it does not require the explicit use of failure detectors (although these are required to solve the Consensus problem). Thus, it is not bound to any particular failure detector mechanism. 


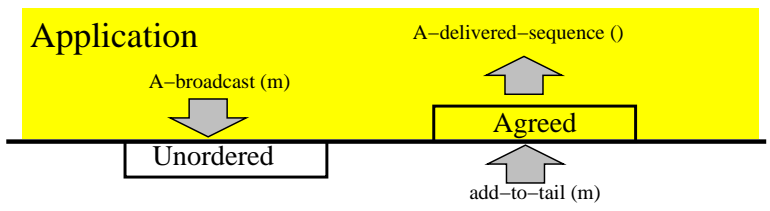

Atomic Broadcast

\begin{tabular}{|c|c|c|c|}
\hline 5 & result & multisend (m) & $\overbrace{2}^{\text {receive (m) }}$ \\
\hline (k, proposed) & 4 & & \\
\hline
\end{tabular}

A_delivered-sequence()

Figure 1: Protocol Interfaces

\section{The Basic Protocol}

\subsection{Basic Principles}

The proposed protocol borrows some of its principles from the total order protocol designed for the crash (no-recovery) model that is described in [3].

As illustrated in Figure 1, the protocol interfaces the upper layer through two variables: the Unordered set and the Agreed queue. Messages requested to be atomically broadcast are added to the Unordered set. Ordered messages are inserted in the Agreed queue, according to their relative order. The Agreed is a representation of the delivery sequence. Operations on the Unordered and Agreed variables must be idempotent, i.e., if the same message is added twice the result is the same as if it is added just once (since message have unique identifiers, duplicates can be detected and eliminated).

The protocol requires the use of a Consensus protocol and of an unreliable (but fair) transport protocol offering the send, multisend, and receive primitives described in Section 3. The transport protocol is used to gossip information among processes. The interface with the Consensus protocol is provided by the propose and decided primitives. The propose primitive accepts two parameters: an integer, identifying a given instance of the Consensus, and a proposed value (a set of messages) for that instance. When a Consensus execution terminates, the decided primitive returns the messages decided by that instance of the Consensus in the variable result. The Consensus primitives must also be idempotent: upon recovery, a process may (re-)invoke these primitives for a Consensus instance that has already started or even terminated.

The Atomic Broadcast protocol works in consecutive rounds. In each round, messages from the Unordered set are proposed to Consensus and the resulting decided messages moved to the Agreed queue. Before proceeding to the next round, the process logs its state in stable storage such that, if it crashes and 
later recovers, it can re-start ordering messages from the last agreed messages.

Processes periodically gossip their round number and their Unordered set of messages to other processes. This mechanism provides the basis for the dissemination of unordered messages among good processes. When a GOSSIP message is received from a process with a lower round number, this means that the sender of the gossip message may have been down and missed the last broadcasts.

\subsection{Protocol Description}

We now provide a more detailed description of the protocol. The protocol is illustrated in Figure 2. The state of each process $p$ is composed of:

- $k_{p}$ : the round counter (initialized to 0 )

- Proposed $_{p}$ : an array of sets of messages proposed to Consensus. Pro$\operatorname{posed}_{p}\left[k_{p}\right]$ is the set of messages proposed to the $k_{p}^{t h}$ Consensus. All entries of the array are initialized to $\perp$ ( $\perp$ means "this entry of the array has not yet been used").

- $\operatorname{Unordered}_{p}$ : a set of unordered messages, requested for broadcast (initialized to $\emptyset$ )

- Agreed $_{p}$ : a queue of already ordered messages (initialized to $\perp$ )

- gossip- $k_{p}$ : a variable that keeps the value of the highest Consensus round known as already decided (this value is obtained via the gossiping mechanism).

The first four variables can be structured as two pairs of variables. The $\left(k_{p}\right.$, Proposed $\left._{p}\right)$ pair is related to the current (and previous) Consensus in which $p$ is (was) involved. The $\left(\right.$ Agreed $_{p}$, Unordered $\left._{p}\right)$ pair is related to the upper layer interface. Statements associated with message receptions are executed atomically. The sequencer task and the gossip task constitute the core of the protocol. Both tasks access atomically the variables $k_{p}$ and Unordered $_{p}$.

A-broadcast $(m)$ issued by process $p$ consists in adding $m$ to its set Unordered $_{p}$. Then the protocol constructs the common delivery order. A-deliver issued by $p$ takes the next message from the Agreed $_{p}$ queue and A-delivers it to the upper layer application. The activation of the protocol is similar in the initial case and in the recovery case: the gossip and sequencer tasks are started (line $a$ ).

The gossip task This task is responsible for disseminating periodically a relevant part of the current state of processes. The gossip message sent by a process $p$, namely $\operatorname{GOSSIP}\left(k_{p}\right.$, Unordered $\left._{p}\right)$, contains its round number and its set of unordered messages. The goal of the gossip task is twofold. In first place, it ensures the dissemination of data messages, such that they are eventually proposed to Consensus by all good processes. In second place, it allows a process that has been down to know which is the most up-to-date round.

Upon reception of a GOSSIP message, an active process $p$ updates its Unordered $_{p}$ set and checks if the sender $q$ has a higher round number $\left(k_{p}>k_{q}\right)$. In this case, $p$ records that it has lagged behind by updating the $g o s s i p-k_{p}$ variable. This variable is used by the sequencer task to get the result of the Consensus $p$ has missed. 


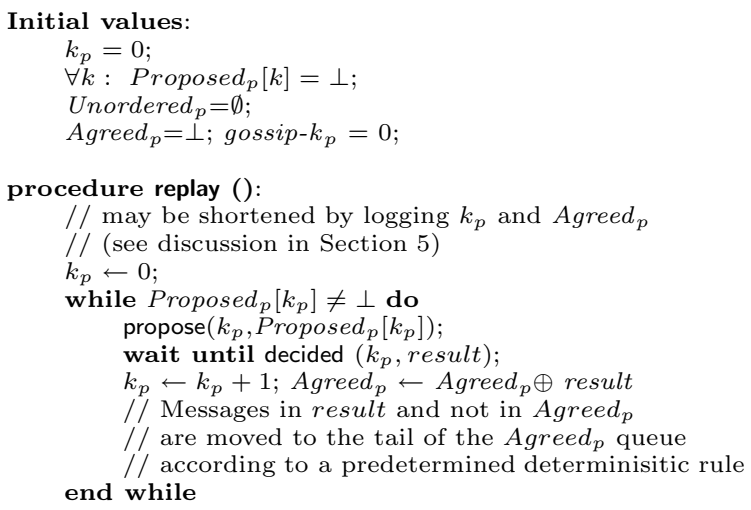

upon initialization or recovery:

retrieve $\left(\right.$ Proposed $\left._{p}\right)$;

replay () ;

(a) fork task $\{$ sequencer and gossip \}

Task gossip:

repeat forever

multisend $\operatorname{GOSSIP}\left(k_{p}\right.$, Unordered $\left._{p}\right)$

upon A-broadcast $(m): / /$ (issued by the upper layer)

Unordered $_{p} \leftarrow$ (Unordered $\left._{p} \cup\{m\}\right) \backslash$ Agreed $_{p}$;

wait until $\left(m \in\right.$ Agreed $_{p}$ ) // see discussion

upon receive $\operatorname{GOSSIP}\left(k_{q}, U_{q}\right)$ from $q$ :

Unordered $_{p} \leftarrow\left(\right.$ Unordered $\left._{p} \cup U_{q}\right) \backslash$ Agreed $_{p}$

if $\left(k_{q}>k_{p}\right)$ then gossip- $k_{p} \leftarrow \max \left(\operatorname{gossip}_{-} k_{p}, k_{q}\right) \mathrm{fi} / / q$ was ahead

Task sequencer:

repeat forever

if $\operatorname{Proposed}_{p}\left[k_{p}\right]=\perp$ then

// Process $p$ has to define its initial value for the next Consensus

wait until $\left(\left(\right.\right.$ Unordered $\left._{p} \neq \emptyset\right)$ or $\left(\right.$ gossip- $\left.\left._{p}>k_{p}\right)\right)$;

Proposed $_{p}\left[k_{p}\right] \leftarrow$ Unordered $_{p}$;

// Ensure that despite crashes

$p$ always proposes the same input to the $k_{p}^{t h}$ Consensus

$\log \left(\operatorname{Proposed}_{p}\left[k_{p}\right]\right)$;

propose $\left(k_{p}\right.$, Proposed $\left._{p}\left[k_{p}\right]\right)$;

fi;

// Actually, the log is done as the first operation of the Consensus (see Section 4.3)

wait until decided $\left(k_{p}\right.$, result $)$;

// Messages in result and not in Agreed $_{p}$ are moved to the tail of

// the Agreed $_{p}$ queue according to a predetermined determinisitic rule

// Initializes the new round and commits results from previous round

$\left[k_{p} \leftarrow k_{p}+1 ;\right.$ Agreed $_{p} \leftarrow$ Agreed $_{p} \oplus$ result ];

end repeat

upon A-delivered-sequence: // (issued by the upper layer)

return Agreed $_{p}$

Figure 2: Atomic Broadcast protocol (Behavior of Process $p$ ) 
The sequencer task This task is the heart of the ordering protocol [3]. The protocol proceeds in rounds. In the round $k$, a process $p$ proposes its $U_{n o r d e r e d}$ set to the $k^{t h}$ instance of Consensus. Before starting the Consensus, the proposed value is saved in stable storage. Note that the propose primitive must be idempotent: in case of crash and recovery, it may be called for the same round more than once. The result of the Consensus is the set of messages to be assigned sequence number $k$. These messages are moved (according to a deterministic rule) from the Unordered $_{p}$ set to the Agreed $_{p}$ queue. Then, the round number $k_{p}$ is incremented and the messages that remain in the Unordered $_{p}$ set are proposed by process $p$ during the next Consensus.

To avoid running unnecessary instances of Consensus, a process does not starts a new round unless it has some messages to propose or it knows it has lagged behind other processes. In the later case, it can propose an empty set as the initial value for those Consensus it has missed (this is because for those Consensus a decision has already been taken without taking $p$ 's proposal into account).

The sequencer task has to execute some statements atomically with respect to the processing of GOSSIP messages. This is indicated by bracketing with "[" and "]" the corresponding statements in the sequencer task.

Logging into stable storage Logging is used to create checkpoints from which a recovering process can continue its execution and consequently make the protocol live. So, at a critical point, the values of relevant variables are logged into stable storage. In this paper we are interested in discussing a protocol that makes a minimal number of ckeckpoints (independently of those required by the underlying Consensus protocols). Thus, we only log the initial value proposed for each Consensus round. This guarantees that if process $p$ crashes before the Consensus decides, $p$ will propose the same value again after recovering. We will later argue that this logging step cannot be avoided.

Note that we do not log the Unordered $p$ set or the Agreed $_{p}$ queue. The Agreed $_{p}$ queue is re-constructed upon recovery from the results of past Consensus rounds by the replay procedure. To ensure that messages proposed to Atomic Broadcast are not lost, the A-broadcast $(m)$ primitive does not returns until the message $m$ is in the agree queue. If the process fails before that, there is no guaranty that the message has been logged, so the message may have or may have not been A-broadcasted. The latter case, it is the same as if the process has failed immediately before calling A-broadcast $(m)$. Note that these design options that aim at minimizing the number of logging operations, do not necessarily provide the more efficient implementation. Alternative designs are discussed below.

Recovery Since the protocol only logs the initial values proposed for each instance of Consensus, the current round $k_{p}$ and the Agreed $_{p}$ queue have to be re-constructed upon recovery. The current round is simply the round for which no initial value has been proposed yet. The agreed queue can be reconstructed by reading the results of the Consensus instances that have terminated. Thus, before forking the sequencer and gossip tasks, the process parses the log of proposed and agreed values (which is kept internally by Consensus). 


\subsection{On the Minimal Logging}

Our solution only requires the logging of the initial proposed value for each round of Consensus. We argue that this logging operation is required for every atomic protocol that uses Consensus as a black box. In fact, all Consensus protocols for the crash-recovery model we are aware of assume that a process $p$ proposes a value by writing it on stable storage. For instance, upon recovery the protocol of [1] checks the stable storage to see if a initial value has been proposed.

\section{An Alternative Protocol}

We now present a number of modifications to our basic protocol that, although increasing slightly the complexity and the number of log operations, may provide some benefits in practical systems. The protocol proposes a state transfer mechanism and additional log operations to reduce the recovery overhead and increase throughput. Additionally, the protocol shows how to prevent the number of entries in the logs from growing indefinitely, by taking application-level checkpoints. These changes are described below. The version of the protocol that takes into account the previous considerations is illustrated in Figure 3 and Figure 4.

\subsection{Avoiding the Replay Phase}

In the previous protocol, we have avoided any logging operation that is not strictly required to ensure protocol correctness. In particular, we have avoided to $\log$ the current round $\left(k_{p}\right)$ and agreed queue $\left(\right.$ Agreed $\left._{p}\right)$, since they can be recomputed from the entries of the array proposed $_{p}$ that have been logged. However, this forces the recovering process to replay the actions taken for each Consensus result (i.e., insert the messages in the agreed queue according to the predetermined deterministic rule).

Faster recovery can be obtained at the expense of periodically checkpointing both variables. The frequency of this checkpointing has no impact on correctness and is an implementation choice (that must weight the cost of checkpointing against the cost of replaying). Note that old proposed values that do are not going to be replayed can be discarded from the log (line $c$ ).

\subsection{Size of logs and application-level checkpoint}

A problem with the current algorithm is that the size of the logs grows indefinitely. A way to circumvent this behavior is to rely on an application-level checkpointing has described below.

In some applications, the state of the application will be determined by the (totally ordered) messages delivered. Thus, instead of logging all the messages, it might be more efficient to log the application state which logically "contains" the Agreed queue. For instance, when the Atomic Broadcast is used to update replicated data, the most recent version of the data can be logged instead of all the past updates. Thus, a checkpoint of the application state can substitute the associated prefix of the delivered message log. 


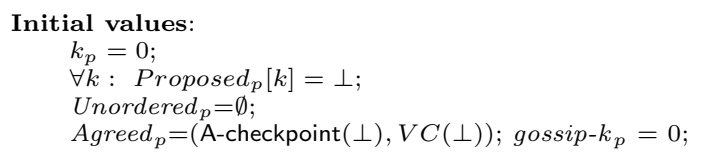

procedure replay ()$: * * * * *$ same as before without its first line $* * * * *$

while $\operatorname{Proposed}_{p}\left[k_{p}\right] \neq \perp$ do

propose $\left(k_{p}\right.$, Proposed $\left._{p}\left[k_{p}\right]\right)$;

wait until decided $\left(k_{p}\right.$, result $)$;

$k_{p} \leftarrow k_{p}+1 ;$ Agreed $_{p} \leftarrow$ Agreed $_{p} \oplus$ result

// Messages in result and not in Agreed $_{p}$ are moved to the tail of

// the Agreed $_{p}$ queue according to a predetermined determinisitic rule

upon initialization or recovery:

**** same as before with the addition of the first line *****

retrieve $\left(k_{p}\right.$, Agreed $\left._{p}\right)$; retrieve $\left(\right.$ Unordered $\left._{p}\right)$;

retrieve $\left(\right.$ Proposed $\left._{p}\right)$;

replay ();

(a) fork task \{ sequencer and gossip and checkpoint \}

upon A-broadcast $(m): / /$ (issued by the upper layer) $* * * * *$ first line: same as before $* * * * *$ Unordered $_{p} \leftarrow$ (Unordered $\left.p \cup\{m\}\right) \backslash$ Agreed $_{p}$;

$\log \left(\right.$ Unordered $\left._{p}\right)$

upon receive $\operatorname{Gossip}\left(k_{q}, U_{q}\right)$ from $q$ : ***** first two lines: same as before $* * * * *$ Unordered $_{p} \leftarrow$ (Unordered $\left.p \cup U_{q}\right) \backslash$ Agreed $_{p}$;

if $\left(k_{q}>k_{p}\right)$ then gossip- $k_{p} \leftarrow \max \left(\right.$ gossip- $\left.k_{p}, k_{q}\right) / / q$ is ahead

(d) else if $\left(k_{p}>k_{q}+\delta\right)$ then

$/ / \delta$ is a configuration parameter

fi fi

send $\operatorname{State}\left(k_{p}-1\right.$, Agreed $\left._{p}\right)$ то $q$

pon receive $\operatorname{STATE}\left(k_{q}, A_{q}\right)$ from $q$ : ***** new message $* * * * *$

if $k_{p}<k_{q}-\delta$ then // $p$ is late

(e) terminate task $\{$ sequencer $\}$;

// Skip Consensus whose number $k$ is such that $k_{p} \leq k<k_{q}$

// so, during the processing of the STATE message, the sequencer task is aborted

$k_{p} \leftarrow k_{q}+1 ;$ Agreed $_{p} \leftarrow A_{q}$;

( $f$ f fork task $\{$ sequencer $\}$ else

fi

gossip- $k_{p} \leftarrow \max \left(\right.$ gossip- $\left.k_{p}, k_{q}\right) / /$ small de-synchronization

Figure 3: Reducing the log size and the number of replay steps (main) 
Task gossip: ***** same as before $* * * * *$

repeat forever multisend $\operatorname{GOSSIP}\left(k_{p}\right.$, Unordered $\left._{p}\right)$

Task checkpoint: ***** new task $* * * * *$

repeat forever // implementation dependent frequency

(b) $\quad\left[\right.$ Agreed $_{p} \leftarrow\left(\right.$ A-checkpoint $\left(\right.$ Agreed $\left._{p}\right), V C\left(\right.$ Agreed $\left.\left.\left._{p}\right)\right)\right]$

$\log \left(k_{p}\right.$, Agreed $\left._{p}\right)$

(c) $\quad / /$ Proposed $_{p}[i], i<k_{p}$ can be discarded from the log

Task sequencer: $* * * * *$ same as before $* * * * *$

repeat forever

if $\operatorname{Proposed}_{p}\left[k_{p}\right]=\perp$ then

// Process $p$ has to define its initial value for the next Consensus

wait until $\left(\left(\right.\right.$ Unordered $\left._{p} \neq \emptyset\right)$ or $\left(\right.$ gossip- $\left.\left._{p}>k_{p}\right)\right)$;

Proposed $_{p}\left[k_{p}\right] \leftarrow$ Unordered $_{p} ;$

// Ensure that despite crashes $p$ always proposes

the same input to the $k_{p}^{t h}$ Consensus

$\log \left(\right.$ Proposed $\left._{p}\left[k_{p}\right]\right)$;

propose $\left(k_{p}\right.$, Proposed $\left._{p}\left[k_{p}\right]\right)$

fi;

// Actually, the log is done as the first operation of the Consensus

wait until decided $\left(k_{p}\right.$, result $)$;

// Messages in result and not in Agreed $_{p}$ are moved to the tail of

// the Agreed $_{p}$ queue according to a predetermined determinisitic rule

// Initializes the new round and commits results from previous round

$\left[k_{p} \leftarrow k_{p}+1 ;\right.$ Agreed $_{p} \leftarrow$ Agreed $_{p} \oplus$ result $] ;$

Unordered $_{p} \leftarrow$ Unordered $_{p} \backslash$ Agreed $_{p}$

end repeat

upon A-deliver-sequence: // (issued by the upper layer) ***** same as before ***** return Agreed $_{p}$

Figure 4: Reducing the log size and the number of replay steps (tasks) 


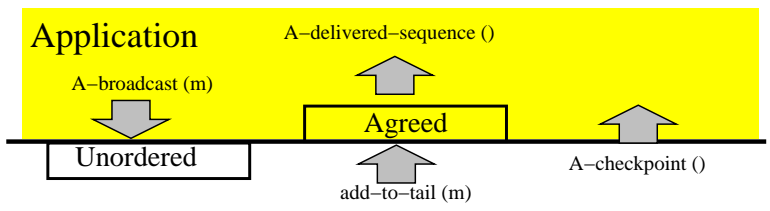

Atomic Broadcast

\begin{tabular}{|c|c|c|c|}
\hline 5 & result & multisend (m) & $\overbrace{2}^{\text {receive (m) }}$ \\
\hline (k, proposed) & 4 & & \\
\hline
\end{tabular}

A_delivered-sequence()

Figure 5: Augmented Protocol Interfaces

In order to exploit this property, one needs to augment the interface with the application layer with an upcall to obtain the application state, as illustrated in Figure 5. The upcall, state $=\mathrm{A}$-checkpoint $\left(\mu_{p}\right)$, accepts as an input parameter a sequence of delivered messages and returns the application state that "contains" those updates. A-checkpoint $(\perp)$ returns the initial state of the application. In order to know which messages are associated with a given checkpoint, a checkpoint vector clock $V C\left(\mu_{p}\right)$ is associated to each checkpoint. The vector clock stores the sequence number of the last message delivered from each process "contained" in the checkpoint. An application-level checkpoint is defined by the pair (A-checkpoint $\left.\left(\mu_{p}\right), V C\left(\mu_{p}\right)\right)$. The sequence of messages delivered to a process is redefined to include an application checkpoint plus the sequence of messages delivered after the checkpoint. A message $m$ belongs to the delivery sequence if it appears explicitly in the sequence or if it is logically included in the application checkpoint that initiates the sequence (this information is preserved by the checkpoint vector clock).

In our protocol, the application state is periodically checkpointed and the delivered messages in the Agreed queue are replaced by the associated applicationlevel checkpoint. This not only offers a shorter replay phase but also prevents the number of entries in the logs from growing indefinitely.

\subsection{State Transfer}

In the basic protocol, a process that has been down becomes aware that it has missed some Consensus rounds when it detects that some other process is already in an higher round of Consensus (through the gossip messages). When this happens, it activates the Consensus instances that it has missed in order to obtain the correspondent agreed messages. A process that has been down for a long period may have missed many Consensus and may require a long time to 
"catch-up".

An alternative design consists in having the most up-to-date process to send a STATE message containing its current round number $k_{p}$ and its Agreed $_{p}$ queue. When a process $p$ that is late receives a STATE message from a process $q$ with a higher round number $\left(k_{p}<k_{q}\right)$ it stops its sequencer task (line $e$ ), updates its state such that it catches up with that process, and re-starts its sequencer task from the updated state (line $f$ ), effectively skipping the Consensus instances it has missed.

Both approaches coexist in the final protocol. A late process can recover by activating the Consensus instances that it has missed or by receiving a STATE message. The amount of de-synchronization that triggers a state transfer can be tuned through the variable $\delta$ (line $d$ ).

Note that, for clarity, we have made the STATE message to carry the complete Agreed queue. Simple optimizations can minimize the amount of state to be transfered. For instance, since the associated GOSSIP messages carries the current round number of the late process, the STATE message can be made to carry only those messages that are not known by the recipient (see $[12,19])$.

\subsection{Sending Message Batches}

For better throughput, it may be interesting to let the application propose batches of messages to the Atomic Broadcast protocol, which are then proposed in batch to a single instance of Consensus. Unfortunately, the definition of Atomic Broadcast implies that every message that has been proposed by a good process be eventually delivered. When there are crashes, a way to ensure this property is not to return from A-broadcast $(m)$ before $m$ is logged. In the basic protocol we wait until the message is ordered (and internally logged by the Consensus). In order to return earlier, the A-broadcast interface needs to log the Unordered $_{p}$ set.

\subsection{Incremental logging}

As described, the protocol emphasizes the control locations where values have to be logged. The actual size of these values can be easily reduced. When logging a queue or a set (such as the Unordered set) only its new part (with respect to the previous logging) has to be logged. This means that a log operation can be saved each time the current value of a variable that has to be logged does not differ from its previously logged value.

\subsection{Correctness of the Alternative Protocol}

As previously, when crashes are definitive, the protocol reduces to the ChandraToueg's Atomic Broadcast protocol [3]. When processes crash and recover, the properties defining the Atomic Broadcast problem (defined in Section 2.2) can be proved by taking into account the following properties ${ }^{3}$ (Due to space limitation, the development of the full proof is omitted).

- (P1) The sequence of consecutive round numbers logged by a process $p$ is

\footnotetext{
${ }^{3}$ These properties actually constitute lemmas of a complete proof. This complete proof bears some ressemblance to the proof described in [11].
} 
not decreasing.

- (P2) If a process $p$ has logged $k_{p}$ whose value is $k$, then its variable $k_{p}$ will always be $\geq k$.

- (P3) If a good process joins round $k$, then all good processes will join a round $\geq k$.

- (P4) For any $k$, independently of the number of times $p$ participates in Consensus numbered $k$, the value it proposes to this Consensus is always the same (despite crashes and despite total/partial Consensus re-executions).

- (P5) For any $k$, independently of the number of times $p$ participates in Consensus numbered $k$, the result value is the same each time the invocation of decided $(k,$.$) terminates at p\left({ }^{4}\right)$. (This property follows from the Consensus specification.)

- (P6) Any message $m$ that has been A-broadcast by a good process will eventually be deposited in Unordered $_{p}$ or Agreed $_{p}$ by any good process $p$.

- (P7) Any message $m$ that has been A-delivered by a process will eventually be deposited in Agreed $_{p}$ by any good process $p$.

The Termination property follows from these properties and from the fact Consensus executions terminate. (So, the Atomic Broadcast protocol is live when the underlying Consensus is live.) The Integrity property follows from The $\oplus$ operation on the Agreed $_{p}$ queue that adds any message $m$ at most once into this queue. The Validity property directly follows from the fact the protocol does not create messages. The Total Order property follows from the use of the underlying Consensus and from the appropriate management of the Agreed $_{p}$ queue.

\section{Related Problems}

\subsection{Consensus vs Atomic Broadcast}

In this paper we have shown how to transform a Consensus protocol for the asynchronous crash-recovery model into an atomic broadcast protocol. It is easy to show that the reduction in the other direction also holds [3]. To propose a value a process atomically broadcasts it; the first value to be delivered can be chosen as the decided value. Thus, both problems are equivalent in asynchronous crash-recovery systems.

\subsection{Atomic Broadcast and Transactional Systems}

It has been shown that a deferred update replication model for fully replication databases can exhibit a better throughput if implemented with an Atomic Broadcast-based termination protocol than if implemented with Atomic Commitment [15]. The idea of the deferred update model is to process the transaction locally and then, at commit time, execute a global certification procedure. The certification phase uses the transaction's read and write sets to detect conflicts with already committed transactions. The use of an Atomic Broadcast primitive ensure that all managers certify transactions in the same order and maintain a

\footnotetext{
${ }^{4}$ Using the terminology used in [3], this means that, after the first Consensus execution numbered $k$, the result value associated with round $k$ is "locked".
} 
consistent state. [15] also proposes designs for Atomic Broadcast protocols in the crash-recovery model but these solutions are not Consensus-based.

\subsection{Atomic Broadcast and Quorum-Based Systems}

In a recent report [18] we show how to extend the Atomic Broadcast primitive to support the implementation of Quorum-based replica management in crashrecovery systems. The proposed technique makes a bridge between established results on Weighted Voting and recent results on the Consensus problem.

\subsection{Total Order Multicast to Distinct Groups}

In this paper we have focused on the Atomic Broadcast problem for a single group of processes. Often, one is required to send messages to more than on group. The problem of efficiently implementing atomic multicast across different groups in crash (no-recovery) asynchronous systems has been solved in several papers $[6,17]$. Since these solutions are based on a Consensus primitive, it is possible to extend them to crash-recovery systems using an approach similar to the one that has been followed here.

\section{Conclusion}

This paper has proposed an Atomic Broadcast primitive for asynchronous crashrecovery distributed systems. Its concept has been based on a building block implementing Consensus. This building block is used as a black box, so our solution is not bound to any particular implementation of Consensus. The protocol is non-blocking in the following sense: as long as the underlying Consensus is live, the Atomic Broadcast protocol does not block good processes despite the behavior of bad processes. Moreover, our solution does not require the explicit use of failure detectors (even though are required to solve the underlying Consensus). Thus, it is not bound to any particular failure detection mechanism. Also, we have shown that Atomic Broadcast can be solved without requiring any additional $\log$ operation in excess of those required by the Consensus. Finally, we have discussed how additional log operations can improve the protocol.

\section{Acknowledgments}

We are grateful to Rachid Guerraoui for his constructive comments on an earlier version of the paper.

\section{References}

[1] M. Aguilera, W. Chen and S. Toueg, Failure Detection and Consensus in the Crash-Recovery Model. Proc. 12th Int. Symposium on DIStributed Computing (formerly WDAG)), Andros, Greece, Sringer-Verlar LNCS 1499, pp. 231-245, September 1998. 
[2] Ö. Babaog̃lu and S. Toueg, Understanding Non-Blocking Atomic Commitement. Chapter 6, Distributed Systems (2nd edition), ACM Press (S. Mullender Ed.), New-York, pp. 147-168, 1993.

[3] T. Chandra and S. Toueg, Unreliable Failure Detectors for Reliable Distributed Systems. Journal of the ACM, 43(2): 225-267, 1996.

[4] D. Dolev , R. Friedman., I. Keidar and D. Malkhi, Failure Detectors in Omission Failure Environments. Proc. 16th Annual ACM Symposium on Principles of Distributed Computing, Santa Barbara, California, page 286, 1997.

[5] M. Fischer, N. Lynch and M. Paterson, Impossibility of Distributed Consensus with One Faulty Process. Journal of ACM, 32(2):374-382, 1985.

[6] U. Fritzke Jr., Ph. Ingels, A. Moustefaoui and M. Raynal, Fault-Tolerant Total Order Multicast To Asynchronous Groups. Proc. 17th IEEE Symposium on Reliable Distributed Systems, West Lafayette (IN), pp. 228-234, October 1998.

[7] J. Gray and A. Reuter, Transaction Processing: Concepts and Techniques. Morgan Kaufmann Pub., 1070 pages, 1993.

[8] R. Guerraoui and A. Schiper, Software-Based Replication for Fault Tolerance. IEEE Computer, 30(4):68-74, 1997.

[9] R. Guerraoui, M. Hurfin, A. Mostefaoui, R. Oliveira, M. Raynal and A. Schiper, Consensus in Asynchronous Distributed Systems: a Concise Guided Tour. Chapter 1: Recent Advances in Large Scale Distributed Systems, Springer-Verlag, LNCS Series (W.G. Broadcast Ed.), To appear 1999.

[10] V. Hadzilacos and S. Toueg, Reliable Broadcast and Related Problems. Chapter 4, Distributed Systems (2nd Edition), ACM Press (S. Mullender Ed.), New-York, pp. 97-145, 1993.

[11] M. Hurfin, A. Mostéfaoui and M. Raynal, Consensus in Asynchronous Systems Where Processes Can Crash and Recover. Proc. 17th IEEE Symposium on Reliable Distributed Systems, West Lafayette (IN), pp. 280-287, October 1998.

[12] R. Ladin, B. Liskov, B. Shrira and S. Ghemawat, Providing Availability Using Lazy Replication. ACM Transactions on Computer Systems, 10(4):360-391, 1992.

[13] N. Lynch, Data Link Protocols. Chapter 16, Distributed Algorithms, Morgan-Kaufmann Pub., pp. 691-732, 1996.

[14] R. Oliveira, R. Guerraoui and A. Schiper, Consensus in the CrashRecovery Model, Research report 97-239, EPFL, Lausanne, Switzerland, 1997.

[15] F. Pedone, R. Guerraoui and A. Schiper, Exploiting Atomic Broadcast in Replicated Databases, Proc. Europar Conference, Springer-Verlag LNCS 1470, pp.513-520, 1998. 
[16] D. Powell (Guest Ed.), Special Issue on Group Communication. Communications of the ACM, 39(4)50-97, 1996.

[17] L. Rodrigues, R. Guerraoui and A. Schiper, Scalable Atomic Multicast. Proc. 7th Int. Conf. on Computer Communications and Networks (IC3N'98), Lafayette (Louisiana), pp. 840-847, October 1998.

[18] L. Rodrigues and M. Raynal, Atomic Broadcast and Quorum-based Replication in Asynchronous Crash-Recovery Distributed Systems. FCUL/DI Technical Report 99-1.

[19] G. Wuu and A. Bernstein, Efficient Solutions to the Replicated Log and Dictionary Problems. Proc. 3rd Int. ACM Symposium on Principles of Distributed Computing (PODC'84), pp. 233-242, 1984. 